\title{
Relations such as Hypernymy: Identifying and Exploiting Hearst Patterns in Distributional Vectors for Lexical Entailment
}

\author{
Stephen Roller \\ Department of Computer Science \\ The University of Texas at Austin \\ rollerecs . utexas. edu
}

\author{
Katrin Erk \\ Department of Linguistics \\ The University of Texas at Austin \\ katrin.erk@mail.utexas.edu
}

\begin{abstract}
We consider the task of predicting lexical entailment using distributional vectors. We perform a novel qualitative analysis of one existing model which was previously shown to only measure the prototypicality of word pairs. We find that the model strongly learns to identify hypernyms using Hearst patterns, which are well known to be predictive of lexical relations. We present a novel model which exploits this behavior as a method of feature extraction in an iterative procedure similar to Principal Component Analysis. Our model combines the extracted features with the strengths of other proposed models in the literature, and matches or outperforms prior work on multiple data sets.
\end{abstract}

\section{Introduction}

As the field of Natural Language Processing has developed, more ambitious semantic tasks are starting to be addressed, such as Question Answering (QA) and Recognizing Textual Entailment (RTE). These systems often depend on the use of lexical resources like WordNet in order to infer entailments for individual words, but these resources are expensive to develop, and always have limited coverage.

To address these issues, many works have considered on how lexical entailments can be derived automatically using distributional semantics. Some focus mostly on the use of unsupervised techniques, and study measures which emphasize particular word relations (Baroni and Lenci, 2011). Many are based on the Distributional Inclusion Hypothesis, which states that the contexts in which a hypernym appears are a superset of its hyponyms' contexts (Zhitomirsky-Geffet and Dagan, 2005; Kotlerman et al., 2010). More recently, a great deal of work has pushed toward using supervised methods (Baroni et al., 2012; Roller et al., 2014; Weeds et al., 2014; Levy et al., 2015; Kruszewski et al., 2015), varying by their experimental setup or proposed model.

Yet the literature disagrees about which models are strongest (Weeds et al., 2014; Roller et al., 2014), or even if they work at all (Levy et al., 2015). Indeed, Levy et al. (2015) showed that two existing lexical entailment models fail to account for similarity between the antecedent and consequent, and conclude that such models are only learning to predict prototypicality: that is, they predict that cat entails animal because animal is usually entailed, and therefore will also predict that sofa entails animal. Yet it remains unclear why such models make for such strong baselines (Weeds et al., 2014; Kruszewski et al., 2015; Levy et al., 2015).

We present a novel qualitative analysis of one prototypicality classifier, giving new insight into why prototypicality classifiers perform strongly in the literature. We find the model overwhelmingly learns to identify hypernyms using Hearst patterns available in the distributional space, like "animals such as cats" and "animals including cats." These patterns have long been used to identify lexical relations (Hearst, 1992; Snow et al., 2004).

We propose a novel model which exploits this behavior as a method of feature extraction, which we call $H$-feature detectors. Using an iterative procedure similar to Principal Component Analysis, our 
model is able to extract and learn using multiple $\mathrm{H}$ feature detectors. Our model also integrates overall word similarity and Distributional Inclusion, bringing together strengths of several models in the literature. Our model matches or outperforms prior work on multiple data sets. The code, data sets, and model predictions are made available for future research. ${ }^{1}$

\section{Background}

Research on lexical entailment using distributional semantics has now spanned more than a decade, and has been approached using both unsupervised (Weeds et al., 2004; Kotlerman et al., 2010; Lenci and Benotto, 2012; Santus, 2013) and supervised techniques (Baroni et al., 2012; Fu et al., 2014; Roller et al., 2014; Weeds et al., 2014; Kruszewski et al., 2015; Levy et al., 2015; Turney and Mohammad, 2015; Santus et al., 2016). Most of the work in unsupervised methods is based on the Distributional Inclusion Hypothesis (Weeds et al., 2004; Zhitomirsky-Geffet and Dagan, 2005), which states that the contexts in which a hypernym appear should be a superset over its hyponyms' contexts.

This work focuses primarily on the supervised works in the literature. Formally, we consider methods which treat lexical entailment as a supervised classification problem, which take as input the distributional vectors for a pair of words, $(H, w)$, and predict on whether the antecedent $w$ entails the consequent $H .^{2}$

One of the earliest supervised approaches was Concat (Baroni et al., 2012). In this work, the concatenation of the pair $\langle H, w\rangle$ was used as input to an off-the-shelf SVM classifier. At the time, it was very successful, but later works noted that it had major problems with lexical memorization (Roller et al., 2014; Weeds et al., 2014; Levy et al., 2015). That is, when the training and test sets were carefully constructed to ensure they were completely disjoint, it performed extremely poorly. Nonetheless, Concat is continually used as a strong baseline in more recent work (Kruszewski et al., 2015).

\footnotetext{
${ }^{1}$ http://github.com/stephenroller/ emnlp2016

${ }^{2}$ We use the notation $w$ and $H$ for word and hypernym. These variables refer to either the lexical items, or their distributional vectors, depending on context.
}

In response to these issues of lexical memorization, alternative models were proposed. Of particular note are the Diff (Fu et al., 2014; Weeds et al., 2014) and Asym classifiers (Roller et al., 2014). The Diff model takes the vector difference $H-w$ as input, while the Asym model uses both the vector difference and the squared vector difference as input. Weeds et al. (2014) found that Concat moderately outperformed Diff, while Roller et al. (2014) found that Asym outperformed Concat. Both Diff and Asym can also be seen as a form of supervised Distributional Inclusion Hypothesis, with the vector difference being analogous to the set-inclusion measures of some unsupervised techniques (Roller et al., 2014). All of these works focused exclusively on hypernymy detection, rather than the more general task of lexical entailment.

Recently, other works have begun to analyze Concat and Diff for their ability to go beyond just hypernymy detection. Vylomova et al. (2016) take an extensive look at Diff's ability to model a wide variety of lexical relations and conclude it is generally robust, and Kruszewski et al. (2015) have success with a neural network model based on the Distributional Inclusion Hypothesis.

On the other hand, Levy et al. (2015) analyze both Concat and Diff in their ability to detect general lexical entailment on five data sets: two consisting of only hypernymy, and three covering a wide variety of other entailing word relations. They find that both Concat and Diff fail, and analytically show that they are learning to predict the prototypicality of the consequent $H$, rather than the relationship between the antecedent and the consequent, and consider this a form of lexical memorization. They propose a new model, Ksim, which addresses their concerns, but lacks any notion of Distributional Inclusion. In particular, they argue for directly including the cosine similarity of $w$ and $H$ as a term in a custom SVM kernel, in order to determine whether $w$ and $H$ are related all. Ultimately, Levy et al. (2015) conclude that distributional vectors may simply be the wrong tool for the job.

\section{Data and Resources}

Prior work on lexical entailment relied on a variety of data sets, each constructed in a different manner. 
We focus on four different data sets, each of which has been used for evaluation in prior work. Two data sets contain only hypernymy relations, and two consider general lexical entailment.

Our first data set is LEDS, the Lexical Entailment Data Set, originally created by Baroni et al. (2012). The data set contains 1385 hyponymhypernym pairs extracted directly from WordNet, forming a set of positive examples. Negative examples were generated by randomly shuffling the original set of 1385 pairs. As such, LEDS only contains examples of hypernymy and random relations.

Another major data set has been BLESS, the Baroni and Lenci (2011) Evaluation of Semantic Spaces. The data set contains annotations of word relations for 200 unambiguous, concrete nouns from 17 broad categories. Each noun is annotated with its co-hyponyms, meronyms, hypernym and some random words. In this work, we treat hypernymy as positive, and other relations as negative.

These two data sets form our hypernymy data sets, but we cannot overstate their important differences: LEDS is balanced, while BLESS contains mostly negative examples; negatives in BLESS include both random pairs and pairs exhibiting other strong semantic relations, while LEDS only contains random pairs. Furthermore, all of the negative examples in LEDS are the same lexical items as the positive items, which has strong implications on the prototypicality argument of Levy et al. (2015).

The next data set we consider is Medical (Levy et al., 2014). This data set contains high quality annotations of subject-verb-object entailments extracted from medical texts, and transformed into noun-noun entailments by argument alignments. The data contains 12,600 annotations, but only 945 positive examples encompassing various relations like hypernymy, meronomy, synonymy and contextonymy. ${ }^{3}$ This makes it one of the most difficult data sets: it is both domain specific and highly unbalanced.

The final data set we consider is TM14, a variation on the SemEval 2012 Shared Task of identifying the degree to which word pairs exhibit various relations. These relationships include a small amount of hypernymy, but also many more uncommon rela-

\footnotetext{
${ }^{3} \mathrm{~A}$ term for entailments that occur in some contexts, but do not cleanly fit in other categories; e.g. hospital entails doctor.
}

tions (agent-object, cause-effect, time-activity, etc). Relationships were binarized into (non-)entailing pairs by Turney and Mohammad (2015). The data set covers 2188 pairs, 1084 of which are entailing.

These two entailment data sets also contain important differences, especially in contrast to the hypernymy data sets. Neither contains any random negative pairs, meaning general semantic similarity measures should be less useful; And both exhibit a variety of non-hypernymy relations, which are less strictly defined and more difficult to model.

\subsection{Distributional Vectors}

In all experiments, we use a standard, count-based, syntactic distributional vector space. We use a corpus composed of the concatenation of Gigaword, Wikipedia, BNC and ukWaC. We preprocess the corpus using Stanford CoreNLP 3.5.2 (Chen and Manning, 2014) for tokenization, lemmatization, POS-tagging and universal dependency parses. We compute a syntactic distributional space for the $250 \mathrm{k}$ most frequent lemmas by counting their dependency neighbors across the corpus. We use only the top $1 \mathrm{M}$ most frequent dependency attachments as contexts. We use CoreNLP's "collapsed dependencies", in which prepositional dependencies are collapsed e.g. "go to the store" emits the tuples (go, prep:to+store) and (store, prep:to ${ }^{-1}+\mathrm{go}$ ). After collecting counts, vectors are transformed using PPMI, SVD reduced to 300 dimensions, and normalized to unit length. The use of collapsed dependencies is very important, as we will see in Section 4, but other parameters are reasonably robust.

\section{Motivating Analysis}

As discussed in Section 2, the Concat classifier is a classifier trained on the concatenation of the word vectors, $\langle H, w\rangle$. As additional background, we first review the findings of Levy et al. (2015), who showed that Concat trained using a linear classifier is only able to capture notions of prototypicality; that is, Concat guesses that (animal, sofa) is a positive example because animal looks like a hypernym.

Formally, a linear classifier like Logistic Regression or Linear SVM learns a decision hyperplane represented by a vector $\hat{p}$. Data points are compared to this plane with the inner product: those above 
the plane (positive inner product) are classified as entailing, and those below as non-entailing. Crucially, since the input features are the concatenation of the pair vectors $\langle H, w\rangle$, the hyperplane $\hat{p}$ vector can be decomposed into separate $H$ and $w$ components. Namely, if we rewrite the decision plane $\hat{p}=\langle\hat{H}, \hat{w}\rangle$, we find that each pair $\langle H, w\rangle$ is classified using:

$$
\begin{aligned}
& \hat{p}^{\top}\langle H, w\rangle \\
& =\langle\hat{H}, \hat{w}\rangle^{\top}\langle H, w\rangle \\
& =\hat{H}^{\top} H+\hat{w}^{\top} w .
\end{aligned}
$$

This analysis shows that, when the hyperplane $\hat{p}$ is evaluated on a novel pair, it lacks any form of direct interaction between $H$ and $w$ like the inner product $H^{\top} w$. Without any interaction terms, the Concat classifier has no way of estimating the relationship between the two words, and instead only makes predictions based on two independent terms, $\hat{H}$ and $\hat{w}$, the prototypicality vectors. Furthermore, the Diff classifier can be analyzed in the same fashion and therefore has the same fatal property.

We agree with this prototypicality interpretation, although we believe it is incomplete: while it places a fundamental ceiling on the performance of these classifiers, it does not explain why others have found them to persist as strong baselines (Weeds et al., 2014; Roller et al., 2014; Kruszewski et al., 2015; Vylomova et al., 2016). To approach this question, we consider a baseline Concat classifier trained using a linear model. This classifier should most strongly exhibit the prototypicality behavior according to Equation 1, making it the best choice for analysis. We first consider the most pessimistic hypothesis: is it only learning to memorize which words are hypernyms at all?

We train the baseline Concat classifier using Logistic Regression on each of the four data sets, and extract the vocabulary words which are most similar to the $\hat{H}$ half of the learned hyperplane $\hat{p}$. If the classifier is only learning to memorize the training data, we would expect items from the data to dominate this list of closest vocabulary terms. Table 1 gives the five most similar words to the learned hyperplane, with bold words appearing directly in the data set.

Interestingly, we notice there are very few bold words at all in the list. In LEDS, we actually see

\begin{tabular}{|llll|}
\hline LEDS & BLESS & Medical & TM14 \\
\hline material & goods & item & sensitiveness \\
structure & lifeform & unlockable & tactility \\
object & item & succor & palate \\
process & equipment & team-up & stiffness \\
activity & herbivore & non-essential & content \\
\hline
\end{tabular}

Table 1: Most similar words to the prototype $\hat{H}$ learned by the Concat model. Bold items appear in the data set.

some hypernyms of data set items that do not even appear in the data set, and the Medical and TM14 words do not even appear related to the content of the data sets. Similar results were also found for Diff and Asym, and both when using Linear SVM and Logistic Regression. These lists cannot explain the success of the prototypicality classifiers in prior work. Instead, we propose an alternative interpretation of the hyperplane: that of a feature detector for hypernyms, or an $\mathrm{H}$-feature detector.

\subsection{H-Feature Detectors}

Recall that distributional vectors are derived from a matrix $M$ containing counts of how often words co-occur with the different syntactic contexts. This co-occurrence matrix is factorized using Singular Value Decomposition, producing both $W$, the ubiquitous word-embedding matrix, and $C$, the contextembedding matrix (Levy and Goldberg, 2014):

$$
M \approx W C^{\top}
$$

Since the word and context embeddings implicitly live in the same vector space (Melamud et al., 2015), we can also compare Concat's hyperplane with the context matrix $C$. Under this interpretation, the Concat model does not learn what words are hypernyms, but rather what contexts or features are indicative of hypernymy. Table 2 shows the syntactic contexts with the highest cosine similarity to the $\hat{H}$ prototype for each of the different data sets.

This view of Concat as an $\mathrm{H}$-feature detector produces a radically different perspective on the classifier's hyperplane. Nearly all of the features learned take the form of Hearst patterns (Hearst, 1992; Snow et al., 2004). The most recognizable and common pattern learned is the "such as" pattern, as in "animals such as cats". These patterns have been well known to be indicative of hypernymy for over two decades. Other interesting pat- 


\begin{tabular}{|ll|}
\hline LEDS & BLESS \\
\hline nmod:such_as+animal & nmod:such_as+submarine \\
acl:relcl+identifiable & nmod:such_as+ship \\
nmod:of ${ }^{-1}+$ determine & nmod:such_as+seal \\
nmod:of ${ }^{-1}+$ categorisation & nmod:such_as+plane \\
compound+many & nmod:such_as+rack \\
nmod:such_as+pot & nmod:such_as+rope \\
\hline Medical & TM14 \\
\hline nmod:such_as+patch & amod+desire \\
nmod:such_as+skin & amod+heighten \\
nmod:including+skin & nsubj ${ }^{-1}+$ disparate \\
nmod:such_as+tooth & nmod:such_as+honey \\
nmod:such_as+feather & nmod:with ${ }^{-1}+$ body \\
nmod:including+finger & nsubj $^{-1}+$ unconstrained \\
\hline
\end{tabular}

Table 2: Most similar contexts to the prototype $\hat{H}$ learned by the Concat model.

terns are the "including" pattern ("animals including cats") and "many" pattern ("many animals"). Although we list only the six most similar context items for the data sets, we find similar contexts continue to dominate the list for the next 30-50 items. Taken together, it is remarkable that the model identified these patterns using only distributional vectors and only the positive/negative example pairs. However, the reader should note these are not true Hearst patterns: Hearst patterns explicitly relate a hypernym and hyponym using an exact pattern match of a single co-occurrence. On the other hand, these $H$-features are aggregate indicators of hypernymy across a large corpus.

These learned features are much more interpretable than those found in the analysis of prior work like Roller et al. (2014) and Levy et al. (2015). Roller et al. (2014) found no signals of H-features in their analysis of one classifier, but their model was focused on bag-of-words distributional vectors, which perform significantly worse on the task. Levy et al. (2015) also performed an analysis of lexical entailment classifiers, and found weak signals like "such" and "of" appearing as prominent contexts in their classifier, giving an early hint of $\mathrm{H}$-feature detectors, but not to such an overwhelming degree as we see in this work. Critically, their analysis focused on a classifier trained on high-dimensional, sparse vectors, rather than focusing on context embeddings as we do. By using these sparse vectors, their model was unable to generalize across simi- lar contexts. Additionally, their model did not make use of collapsed dependencies, making features like "such" much weaker signals of entailment and therefore less dominant during analysis.

Among these remarkable lists, the LEDS and TM14 data sets stand out for having much fewer "such as" patterns compared to BLESS and Medical. The reason for this is explained by the construction of the data sets: since LEDS contains the same words used as both positive and negative examples, the classifier has a hard time picking out clear signal. The TM14 data set, however, does not contain any such negative examples.

We hypothesize the TM14 data set contains too many diverse and mutually exclusive forms of lexical entailment, like instrument-goal (e.g. "honey" $\rightarrow$ "sweetness"). To test this, we retrained the model with only hypernymy as positive examples, and all other relations as negative. We find that "such as" type patterns become top features, but also some interesting data specific features, like "retailer of [clothes]". Examining the data shows it contains many consumer goods, like "beverage" or "clothes", which explains these features.

\section{Proposed Model}

As we saw in the previous section, Concat only acts as a sort of $\mathrm{H}$-feature detector for whether $H$ is a prototypical hypernym, but does not actually infer the relationship between $H$ and $w$. Nonetheless, this is powerful behavior which should still be used in combination with the insights of other models like Ksim and Asym. To this end, we propose a novel model which exploits Concat's H-feature detector behavior, extends its modeling power, and adds two other types of evidence proposed in the literature: overall similarity, and distributional inclusion.

Our model works through an iterative procedure similar to Principal Component Analysis (PCA). Each iteration repeatedly trains a Concat classifier under the assumption that it acts as an $\mathrm{H}$-feature detector, and then explicitly discards this information from the distributional vectors. By training a new $\mathrm{H}$-feature detector on these modified distributional vectors, we can find additional features indicative of entailment which were missed by the first classifier. The entire procedure is iteratively repeated similar 


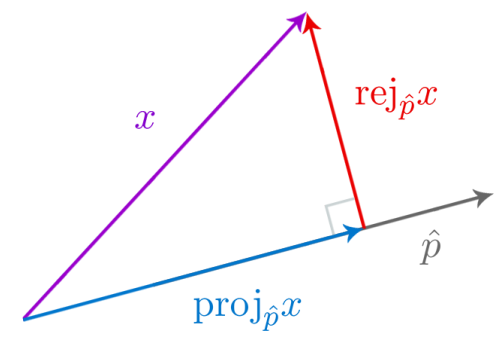

Figure 1: A vector $\hat{p}$ is used to break $x$ into two orthogonal components, its projection and the rejection over $\hat{p}$.

to how in Principal Component Analysis, the second principal component is computed after the first principal component has been removed from the data.

The main insight is that after training some $\mathrm{H}$ feature detector using Concat, we can remove this prototype from the distributional vectors through the use of vector projection. Formally, the vector projection of $x$ onto a vector $\hat{p}, \operatorname{proj}_{\hat{p}}(x)$ finds the component of $x$ which is in the direction of $\hat{p}$,

$$
\operatorname{proj}_{\hat{p}}(x)=\left(\frac{x^{\top} \hat{p}}{\|\hat{p}\|}\right) \hat{p} .
$$

Figure 1 gives a geometric illustration of the vector projection. If $x$ forms the hypotenuse of a right triangle, $\operatorname{proj}_{\hat{p}}(x)$ forms a leg of the triangle. This also gives rise to the vector rejection, which is the vector forming the third leg of the triangle. The vector rejection is orthogonal to the projection, and intuitively, is the original vector after the projection has been removed:

$$
\operatorname{rej}_{\hat{p}}(x)=x-\operatorname{proj}_{\hat{p}}(x) .
$$

Using the vector rejection, we take a learned $\mathrm{H}-$ feature detector $\hat{p}$, and discard these features from each of the word vectors. That is, for every data point $\langle H, w\rangle$, we replace it by its vector rejection and rescale it to unit magnitude:

$$
\begin{aligned}
H_{i+1} & =\operatorname{rej}_{\hat{p}}(H) /\left\|\operatorname{rej}_{\hat{p}}(H)\right\| \\
w_{i+1} & =\operatorname{rej}_{\hat{p}}(w) /\left\|\operatorname{rej}_{\hat{p}}(w)\right\|
\end{aligned}
$$

A new classifier trained on the $\left\langle H_{i+1}, w_{i+1}\right\rangle$ data must now learn a different decision plane than $\hat{p}$, as $\hat{p}$ is no longer present in any data points. This repetition of the procedure is roughly analogous to learning the second principal component of the data; we wish to classify the pairs without using any information learned from the previous iteration.

This second classifier must perform strictly worse than the original, otherwise the first classifier would have learned this second hyperplane. Nonetheless, it will be able to learn new $\mathrm{H}$-feature detectors which the original classifier was unable to capture. By repeating this process, we can find several $\mathrm{H}$ feature detectors, $\hat{p}_{1}, \ldots, \hat{p}_{n}$. Although the first, $\hat{p}_{1}$ is the best possible single $\mathrm{H}$-feature detector, each additional $\mathrm{H}$-feature detector increases the model's representational power (albeit with diminishing returns).

This procedure alone does not address the main concern of Levy et al. (2015): that these linear classifiers never actually model any connection between $H$ and $w$. To address this, we explicitly compare $H$ and $w$ by extracting additional information about how $H$ and $w$ interact with respect to each of the $\mathrm{H}$-feature detectors. This additional information is then used to train one final classifier which makes the final prediction.

Concretely, in each iteration $i$ of the procedure, we generate a four-valued feature vector $F_{i}$, based on the $\mathrm{H}$-feature detector $\hat{p}_{i}$. Each feature vector contains (1) the similarity of $H_{i}$ and $w_{i}$ (before projection); (2) the feature $\hat{p}_{i}$ applied to $H_{i}$; (3) the $\mathrm{H}$ feature detector $\hat{p}_{i}$ applied to $w_{i}$; and (4) the difference of 2 and 3.

$$
\begin{aligned}
& F_{i}\left(\left\langle H_{i}, w_{i}\right\rangle, \hat{p}_{i}\right) \\
& \quad=\left\langle H_{i}^{\top} w_{i}, H_{i}^{\top} \hat{p}_{i}, w_{i}^{\top} \hat{p}_{i},\left(H_{i}-w_{i}\right)^{\top} \hat{p}_{i}\right\rangle
\end{aligned}
$$

These four "meta"-features capture all the benefits of the H-feature detector (slots 2 and 3), while still addressing Concat's issues with similarity arguments (slot 1) and distributional inclusion (slot 4). The final feature's relation to the DIH comes from the observation of Roller et al. (2014) that the vector difference intuitively captures whether the hypernym includes the hyponym.

The union of all the feature vectors $F_{1}, \ldots, F_{n}$ from repeated iteration form a $4 n$-dimensional feature vector which we use as input to one final classifier which makes the ultimate decision. This classifier is trained on the same training data as each of the individual $\mathrm{H}$-feature detectors, so our iterative procedure acts only as a method of feature extraction. 
For our final classifier, we use an SVM with an RBF-kernel, though decision trees and other nonlinear classifiers also perform reasonably well. The nonlinear final classifier can be understood as doing a form of logical reasoning about the four slots: "animal" is a hypernym of "cat" because (1) they are similar words where (2) animal looks like a hypernym, but (3) cat does not, and (4) some "animal" contexts are not good "cat" contexts.

\section{Experimental Setup and Evaluation}

In our experiments, we use a variation of 20 -fold cross validation which accounts for lexical overlap. To simplify explanation, we first explain how we generate splits for training/testing, and then afterwards introduce validation methodology.

We first pool all the words from the antecedent (LHS) side of the data into a set, and split these lexical items into 20 distinct cross-validation folds. For each fold $F_{i}$, we then use all pairs $(w, H)$ where $w \in F_{i}$ as the test set pairs. That is, if "car" is in the test set fold, then "car $\rightarrow$ vehicle" and "car $\nrightarrow$ truck" will appear as test set pairs. The training set will then be every pair which does not contain any overlap with the test set; e.g. the training set will be all pairs which do not contain "car", "truck" or "vehicle" as either the antecedent or consequent. This ensures that both (1) there is zero lexical overlap between training and testing and (2) every pair is used as an item in a test fold exactly once. One quirk of this setup is that all test sets are approximately the same size, but training sizes vary dramatically.

This setup differs from those of previous works like Kruszewski et al. (2015) and Levy et al. (2015), who both use single, fixed train/test/val sets without lexical overlap. We find our setup has several advantages over fixed sets. First, we find there can be considerable variance if the train/test set is regenerated with a different random seed, indicating that multiple trials are necessary. Second, fixed setups consistently discard roughly half the data as ineligible for either training or test, as lexical items appear in many pairs. Our CV-like setup allows us to evaluate performance over every item in the data set exactly once, making a much more efficient and representative use of the original data set.

Our performance metric is F1 score. This is more

\begin{tabular}{|l|ccrc|}
\hline Model & LEDS & BLESS & Medical & TM14 \\
\hline \hline \multicolumn{5}{|c|}{ Linear Models } \\
\hline Cosine & .787 & .208 & .168 & .676 \\
Concat & .794 & .612 & .218 & .693 \\
Diff & .805 & .440 & .195 & .665 \\
Asym & .865 & .510 & .210 & .671 \\
Concat+Diff & .801 & .604 & .224 & .703 \\
Concat+Asym & .843 & $\mathbf{. 6 3 1}$ & .240 & .701 \\
\hline \multicolumn{5}{|c|}{ Nonlinear Models } \\
\hline RBF & .779 & .574 & .215 & .705 \\
Ksim & .893 & .488 & .224 &. $\mathbf{7 0 7}$ \\
Our model & $\mathbf{. 9 0 1}$ & $\mathbf{. 6 3 1}$ & $\mathbf{. 2 6 0}$ & .697 \\
\hline
\end{tabular}

Table 3: Mean F1 scores for each model and data set.

representative than accuracy, as most of the data sets are heavily unbalanced. We report the mean F1 scores across all cross validation folds.

\subsection{Hyperparameter Optimization}

In order to handle hyperparameter selection, we actually generate the test set using fold $i$, and use fold $i-1$ as a validation set (removing pairs which would overlap with test), and the remaining 18 folds as training (removing pairs which would overlap with test or validation). We select hyperparameters using grid search. For all models, we optimize over the regularization parameter $C \in$ $\left\{10^{-4}, 10^{-3}, \ldots, 10^{4}\right\}$, and for our proposed model, the number of iterations $n \in\{1, \ldots, 6\}$. All other hyperparameters are left as defaults provided by Scikit-Learn (Pedregosa et al., 2011), except for using balanced class weights. Without balanced class weights, several of the baseline models learn degenerate functions (e.g. always guess non-entailing).

\section{Results}

We compare our proposed model to several existing and alternative baselines from the literature. Namely, we include a baseline Cosine classifier, which only learns a threshold which maximizes F1 score on the training set; three linear models of prior work, Concat, Diff and Asym; and the RBF and Ksim models found to be successful in Kruszewski et al. (2015) and Levy et al. (2015). We also include two additional novel baselines, Concat+Diff and Concat+Asym, which add a notion of Distributional Inclusion into the Concat baseline, but are still linear models. We cannot include baselines like 


\begin{tabular}{|l|rrrr|}
\hline Model & LEDS & BLESS & Medical & TM14 \\
\hline No Similarity & .099 & .061 & .034 & .003 \\
No Detectors & -.008 & .136 & .018 & .028 \\
No Inclusion & .010 & .031 & .014 & .001 \\
\hline
\end{tabular}

Table 4: Absolute decrease in mean F1 on the development sets with the different feature types ablated. Higher numbers indicate greater feature importance.

Ksim+Asym, because Ksim is based on a custom SVM kernel which is not amenable to combinations.

Table 3 the results across all four data sets for all of the listed models. Our proposed model improves significantly ${ }^{4}$ over Concat in the LEDS, BLESS and Medical data sets, indicating the benefits of combining these aspects of similarity and distributional inclusion with the $\mathrm{H}$-feature detectors of Concat. The Concat+Asym classifier also improves over the Concat baseline, further emphasizing these benefits. Our model performs approximately the same as Ksim on the LEDS and TM14 data sets (no significant difference), while significantly outperforming it on BLESS and Medical data sets.

\subsection{Ablation Experiments}

In order to evaluate how important each of the various $F$ features are to the model, we also performed an ablation experiment where the classifier is not given the similarity (slot 1), prototype H-feature detectors (slots 2 and 3 ) or the inclusion features (slot 4). To evaluate the importance of these features, we fix the regularization parameter at $C=1$, and train all ablated classifiers on each training fold with number of iterations $n=1, \ldots, 6$. Table 4 shows the decrease (absolute difference) in performance between the full and ablated models on the development sets, so higher numbers indicate greater feature importance.

We find the similarity feature is extremely important in the LEDS, BLESS and Medical data sets, therefore reinforcing the findings of Levy et al. (2015). The similarity feature is especially important in the LEDS and BLESS data sets, where negative examples include many random pairs. The detector features are moderately important for the Medical and TM14 data sets, and critically important on BLESS, where we found the strongest evi-

\footnotetext{
${ }^{4}$ Bootstrap test, $p<.01$.
}

dence of Hearst patterns in the H-feature detectors. Surprisingly, the detector features are moderately detrimental on the LEDS data set, though this can also be understood in the data set's construction: since the negative examples are randomly shuffled positive examples, the same detector signal will appear in both positive and negative examples. Finally, we find the model performs somewhat robustly without the inclusion feature, but still is moderately impactful on three of the four data sets, lending further evidence to the Distributional Inclusion Hypothesis. In general, we find all three components are valuable sources of information for identifying hypernymy and lexical entailment.

\subsection{Analysis by Number of Iterations}

In order to evaluate how the iterative feature extraction affects model performance, we fix the regularization parameter at $C=1$, and train our model fixing the number of iterations to $n=\{1, \ldots, 6\}$. We then measure the mean F1 score across the development folds and compare to a baseline which uses only one iteration. Figure 2 shows these results across all four data sets, with the 0 line set at performance of the $n=1$ baseline. Models above 0 benefit from the additional iterations, while models below do not.

In the figure, we see that the iterative procedure moderately improves performance LEDS, while greatly improving the scores of BLESS and TM14, but on the medical data set, additional iterations actually hurt performance. The differing curves indicate that the optimal number of iterations is very data set specific, and provides differing amounts of improvement, and therefore should be tuned carefully. The LEDS and BLESS curves indicate a sort of "sweet spot" behavior, where further iterations degrade performance.

To gain some additional insight into what is captured by the various iterations of the feature extraction procedure, we repeat the procedure from Section 4: we train our model on the entire BLESS data set using a fixed four iterations and regularization parameter. For each iteration, we compare its learned $\mathrm{H}$-feature detector to the context embeddings, and report the most similar contexts for each iteration in Table 5.

The first iteration is identical to the one in Ta- 


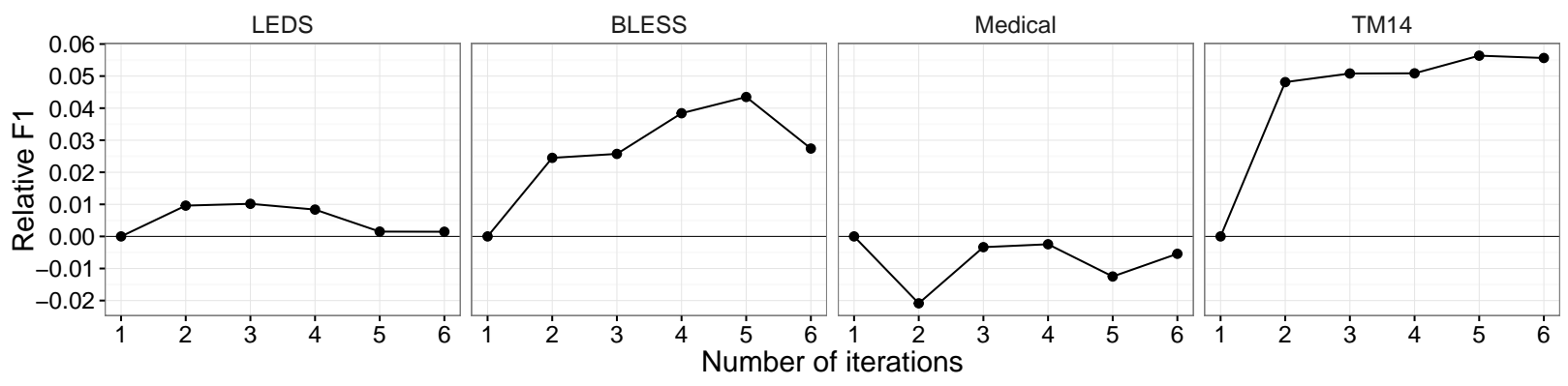

Figure 2: Performance of model on development folds by number of iterations. Plots show the improvement (absolute difference) in mean F1 over the model fixed at one iteration.

\begin{tabular}{|llll|}
\hline Iteration 1 & Iteration 2 & Iteration 3 & Iteration 4 \\
\hline nmod:such_as+submarine & nmod:including+animal & amod+free-swimming & advcl+crown \\
nmod:such_as+ship & nmod:including+snail & nmod:including $^{-1}+$ thing & advcl+victorious \\
nmod:such_as+seal & nmod:including+insect & nsubj $^{-1}+$ scarcer & nsubj+eaters \\
nmod:such_as+plane & nmod:such_as+crustacean & nsubj $^{-1}+$ pupate & nsubj+kaine \\
nmod:such_as+rack & nmod:such_as+mollusc & nmod:such_as+mollusc & nmod:at+finale \\
nmod:such_as+rope & nmod:such_as+insect & nmod:of ${ }^{-1}+$ value & nsubj+gowen \\
nmod:such_as+box & nmod:such_as+animal & nmod:as $^{-1}+$ exhibit & nsubj+pillman \\
\hline
\end{tabular}

Table 5: Most similar contexts to the H-feature detector for each iteration of the PCA-like procedure. This model was trained on all data of BLESS. The first and second iterations contain clear Hearst patterns, while the third and fourth contain some data-specific and non-obvious signals.

ble 2, as expected. The second iteration includes many $\mathrm{H}$-features not picked up by the first iteration, mostly those of the form " $\mathrm{X}$ including $\mathrm{Y}$ ". The third iteration picks up some data set specific signal, like "free-swimming [animal]" and "value of [computer]", and so on. By the fourth iteration, the features no longer exhibit any obvious Hearst patterns, perhaps exceeding the sweet spot we observed in Figure 2. Nonetheless, we see how multiple iterations of the procedure allows our model to capture many more useful features than a single Concat classifier on its own.

\section{Conclusion}

We considered the task of detecting lexical entailment using distributional vectors of word meaning. Motivated by the fact that the Concat classifier acts as a strong baseline in the literature, we proposed a novel interpretation of the model's hyperplane. We found the Concat classifier overwhelmingly acted as a feature detector which automatically identifies Hearst Patterns in the distributional vectors.

We proposed a novel model that embraces these
H-feature detectors fully, and extends their modeling power through an iterative procedure similar to Principal Component Analysis. In each iteration of the procedure, an H-feature detector is learned, and then removed from the data, allowing us to identify several different kinds of Hearst Patterns in the data. Our final model combines these H-feature detectors with measurements of general similarity and Distributional Inclusion, in order to integrate the strengths of different models in prior work. Our model matches or exceeds the performance of prior work, both on hypernymy detection and general lexical entailment.

\section{Acknowledgments}

The authors would like to thank I. Beltagy, Vered Shwartz, Subhashini Venugopalan, and the reviewers for their helpful comments and suggestions. This research was supported by the NSF grant IIS 1523637. We acknowledge the Texas Advanced Computing Center for providing grid resources that contributed to these results. 


\section{References}

Marco Baroni and Alessandro Lenci. 2011. How we BLESSed distributional semantic evaluation. In Proceedings of the GEMS 2011 Workshop on GEometrical Models of Natural Language Semantics, pages 1-10, Edinburgh, UK.

Marco Baroni, Raffaella Bernardi, Ngoc-Quynh Do, and Chung-chieh Shan. 2012. Entailment above the word level in distributional semantics. In Proceedings of the 2012 Conference of the European Chapter of the Association for Computational Linguists, pages 23-32, Avignon, France.

Danqi Chen and Christopher Manning. 2014. A fast and accurate dependency parser using neural networks. In Proceedings of the 2014 Conference on Empirical Methods in Natural Language Processing, pages 740750, Doha, Qatar.

Ruiji Fu, Jiang Guo, Bing Qin, Wanxiang Che, Haifeng Wang, and Ting Liu. 2014. Learning semantic hierarchies via word embeddings. In Proceedings of the 2014 Annual Meeting of the Association for Computational Linguistics, pages 1199-1209, Baltimore, Maryland.

Marti A Hearst. 1992. Automatic acquisition of hyponyms from large text corpora. In Proceedings of the 1992 Conference on Computational Linguistics, pages 539-545, Nantes, France.

Lili Kotlerman, Ido Dagan, Idan Szpektor, and Maayan Zhitomirsky-Geffet. 2010. Directional distributional similarity for lexical inference. Natural Language Engineering, 16:359-389, 10.

Germán Kruszewski, Denis Paperno, and Marco Baroni. 2015. Deriving Boolean structures from distributional vectors. Transactions of the Association for Computational Linguistics, 3:375-388.

Alessandro Lenci and Giulia Benotto. 2012. Identifying hypernyms in distributional semantic spaces. In The First Joint Conference on Lexical and Computational Semantics, pages 75-79, Montréal, Canada.

Omer Levy and Yoav Goldberg. 2014. Neural word embedding as implicit matrix factorization. In $\mathrm{Ad}$ vances in Neural Information Processing Systems, pages 2177-2185.

Omer Levy, Ido Dagan, and Jacob Goldberger. 2014. Focused entailment graphs for Open IE propositions. In Proceedings of the 2014 Conference on Computational Natural Language Learning, pages 87-97, Ann Arbor, Michigan.

Omer Levy, Steffen Remus, Chris Biemann, and Ido Dagan. 2015. Do supervised distributional methods really learn lexical inference relations? In Proceedings of the 2015 North American Chapter of the Association for Computational Linguistics: Human Language Technologies, pages 970-976, Denver, Colorado.
Oren Melamud, Omer Levy, and Ido Dagan. 2015. A simple word embedding model for lexical substitution. In Proceedings of the First Workshop on Vector Space Modeling for Natural Language Processing, pages 17, Denver, Colorado.

F. Pedregosa, G. Varoquaux, A. Gramfort, V. Michel, B. Thirion, O. Grisel, M. Blondel, P. Prettenhofer, R. Weiss, V. Dubourg, J. Vanderplas, A. Passos, D. Cournapeau, M. Brucher, M. Perrot, and E. Duchesnay. 2011. Scikit-learn: Machine learning in Python. Journal of Machine Learning Research, 12:28252830.

Stephen Roller, Katrin Erk, and Gemma Boleda. 2014. Inclusive yet selective: Supervised distributional hypernymy detection. In Proceedings of the 2014 International Conference on Computational Linguistics, pages 1025-1036, Dublin, Ireland.

Enrico Santus, Alessandro Lenci, Tin-Shing Chiu, Qin Lu, and Chu-Ren Huang. 2016. Nine features in a random forest to learn taxonomical semantic relations. In Proceedings of the Tenth International Conference on Language Resources and Evaluation, Paris, France.

Enrico Santus. 2013. SLQS: An entropy measure. Master's thesis, University of Pisa.

Rion Snow, Daniel Jurafsky, and Andrew Y Ng. 2004. Learning syntactic patterns for automatic hypernym discovery. In Advances in Neural Information Processing Systems, pages 1297-1304.

Peter D Turney and Saif M Mohammad. 2015. Experiments with three approaches to recognizing lexical entailment. Natural Language Engineering, 21(03):437-476.

Ekaterina Vylomova, Laura Rimell, Trevor Cohn, and Timothy Baldwin. 2016. Take and took, gaggle and goose, book and read: Evaluating the utility of vector differences for lexical relation learning. In Proceedings of the 54th Annual Meeting of the Association for Computational Linguistics, pages 1671-1682, Berlin, Germany, August.

Julie Weeds, David Weir, and Diana McCarthy. 2004. Characterising measures of lexical distributional similarity. In Proceedings of the 2004 International Conference on Computational Linguistics, pages 10151021, Geneva, Switzerland.

Julie Weeds, Daoud Clarke, Jeremy Reffin, David Weir, and Bill Keller. 2014. Learning to distinguish hypernyms and co-hyponyms. In Proceedings of the 2014 International Conference on Computational Linguistics, pages 2249-2259, Dublin, Ireland.

Maayan Zhitomirsky-Geffet and Ido Dagan. 2005. The distributional inclusion hypotheses and lexical entailment. In Proceedings of the 2005 Annual Meeting of the Association for Computational Linguistics, pages 107-114, Ann Arbor, Michigan. 